\title{
L'Etat dans l'Etat
}

\section{Rainer M. Kaelin}

Dr méd., Spécialiste en médecine interne et Pneumologie, ancien Vice-président de la Ligue pulmonaire suisse et vaudoise, Vice-président de OxyRomandie/ OxySuisse, membre FMH

L'instrumentalisation de la Suisse par le cigarettier Philip Morris (PM), qui s'était assuré le prestige du parrain principal du pavillon suisse à l'Exposition universelle à Dubai en 2020, pose la question, dans quelle mesure les décideurs politiques dans notre pays fixent à l'économie les conditions cadre. Ou bien, si ce n'est plutôt elle qui décide de la politique.

A l'Université de Lausanne a été présenté la première description des relations des cigarettiers avec science et politique suisses, sous forme de thèse de doctorat en médecine-biologie [1]. La Loi sur les produits du tabac (LPTab) a mis ces relations en pleine lumière. Elles expliquent l'absence d'interdictions de la publicité, la loi lacunaire sur la fumée passive, et le refus du Parlement de ratifier la convention cadre de l'OMS et de la mettre en œuvre.

\section{Les structures paraétatiques des groupes d'intérêts}

Les groupes d'intérêt économiques se sont organisés très tôt dans l'Etat fédéral libéral. Ils étaient et sont importants, car la Confédération dispose de moyens limités. Leur influence grandissait avec leur présence sur l'ensemble du territoire national. De ce fait, ils sont aussi appelés "structures paraétatiques» [2]. L'ASFC (Association suisse des fabricants de cigarettes, puis CISC, la Communauté de l'industrie suisse de la cigarette, depuis 2004 «Swiss Cigarette») s'est constitué en 1933. Sa présidence était confié de 1936-2004 à quatre conseillers nationaux qui y accédaient après leurs élections au Parlement. L'importance que l'AFSC attribuait à cette représentation, apparaissait au plein jour, lors que le conseiller national Corbat échoua à sa réélection en 1979 et devait démissionner [3]. Car avec le siège au Parlement on accède aux canaux d'influence: les commissions, les consultations sur les projets de loi, le contact avec l'administration, l'autorégulation, l'influence sur d'autres parlementaires, sur opinion publique et média.

En Suisse, la "phase préparlementaire» du processus législatif est décisive, car les compromis sont négociés dans les commissions bien avant que les lois soient débattues dans le Parlement [4]. Les groupes d'intérêt aux finances solides, comme Swiss Cigarette, regroupant les firmes multinationales PM, British American Tobacco (BAT) et Japan Tobacco international (JTI), disposent d'un moyen de pression supplémentaire, le referendum.

Les limites des commissions extraparlementaires de négocier des compromis se montraient lors que la commission pour la prévention du tabagisme (19871996) fut dissolue par la conseillère fédérale Ruth Dreyfus, car aucun consensus n'était plus possible. Car l'AFSC avait réussi, en court-circuitant l'OFSP, à obtenir «une composition équilibrée, non-biaisée de la commission" avec des sièges pour «des représentants de chacune des industries de la cigarette, des cigares, du tabac de pipe, des planteurs de tabac, des marchands de tabac, et deux représentants de l'industrie de la publicité» [5].

\section{Autorégulation: protection de la jeunesse par la commission de loyauté?}

Les groupes d'intérêt préfèrent l'autorégulation. Sans lois, elle permet d'introduire des solutions et par cela d'éviter la politisation du problème, ce qui mettrait à jour les conflits d'intérêts. L'autorégulation est utilisée pour prouver que des lois sont inutiles et pour se présenter comme élément responsable de la société. Ainsi, depuis 1955 l'industrie du tabac s'est donnée (ou a modifié) six fois ses propres règles concernant la publicité, la dernière fois en 2005 par la convention avec la commission de loyauté (CL). En 1992 ceci fut le cas sous la pression de l'initiative jumelle (interdiction de la publicité pour le tabac et l'alcool). Le vice-président de PM notait alors: «Le but du code est une protection accrue de la jeunesse, en vue du débat parlementaire sur l'initiative jumelle. Les objectifs de l'industrie et de ses alliés sont: 1 . Convaincre les parlementaires 
qu'un contre-projet est inutile et que la protection de la jeunesse se fait mieux par autorégulation... et 2 . Influencer le peuple, afin qu'il rejette l'initiative à cause de son inutilité. Pour obtenir une crédibilité maximale, le code sera contre-signé par 'Publicité Suisse' et son application sera confiée à la commission de loyauté» [6]. En rejetant l'initiative, le Parlement instaura l'autocontrôle. Celle-ci se donna un statut officiel en nommant des politiciens et réseauta par cela les cigarettiers avec les politiques et les professionnels de la publicité. La convention de la CL fut signée par Doris Leuthard, sa présidente d'alors. Président des professionnels de la publicité fut dès 2007 le conseiller aux Etats Carlo Schmid, ancien président du PDC, auquel succéda Filippo Lombardi, conseiller aux Etats et actuel chef de fraction PDC. Présidente de CL est la conseillère nationale Christine BullardMarbach (PDC). Quoique la CL se déclare indépendante, elle ne peut pas l'être [7]: Contrairement aux plaintes concernant d'autres secteurs, une infraction confirmée par elle du secteur tabac ne sera suivie d'aucune sanction, mais uniquement transmise à... Swiss Cigarette [8]. La convergence des intérêts entre les branches du tabac et publicitaire est ainsi renforcée. Elle se reflète également par la structure de l'association "Communication Suisse» [9]. L'industrie du tabac y est représentée à tous les niveaux: en tant que mandataire des agences de relations publiques, en tant qu'auteur de publicité pour ses produits, en tant qu'arbitre du secteur publicitaire. La protection de la jeunesse n'est pas un souci de la CL. Ceci est illustré par le vote prépondérant de sa présidente relatif à une plainte de l'Association Suisse des Enseignants [10]. Contrairement à eux, la CL ne trouvait rien à critiquer à une action de publicité de Mediamarkt, commerce qui proposait des récompenses financières aux élèves pour leur notes à l'école, afin de se rendre populaire dans ce segment de la population [10].

L'administration également se base sur l'autocontrôle. Lors qu'en décembre 2018 l'e-cigarette JUUL causa en Suisse une épidémie de dépendants à la nicotine parmi les jeunes, car sa vente n'était entravée par aucune décision des autorités, le Conseil fédéral convoqua les commerçants à une "table ronde». Le résultat était un «Code» qui ressemble à une action de relations publiques: Les partenaires de la "Swiss Vape Association» (www.svta.ch/kodex) s'engagent volontairement à ne pas vendre les cigarettes électroniques aux moins de 18 ans et de n'adresser à eux aucune publicité. Ils promettaient de s'imposer ces règles, mais il n'est question ni d'une instance d'arbitrage, ni d'une restriction de la promotion ou du parrainage.

\section{Réseautage industrie-politique}

Livre d'or informatif de la firme Burrus: le président de la Confédération Honegger en 1982, le ministre de l'Economie Delamuraz en 1988, les responsables de Coop et de Denner, des banques, de Ringier et Co., du Tages-Anzeiger et autres remercient pour l'accueil amical et chaleureux. Les archives documentent des ententes avec les conseillers d'Etats de Neuchâtel J. Cavadini, et J. Béguin en 1982. Dans le "Corporate Affairs Plan 1987» de PM, on peut lire: «Recruter plus de membres pour le comité du parti du tabac de 21 membres et inciter ceux-ci de communiquer de manière convaincante les vues de l'industrie au parlement. Intégrer ces alliés dans le système d'alarme précoce» [12].

L'argent entretient les relations: «... nous nous devons dès lors de faire appel à une participation sensiblement plus importante de votre part.» La lettre du Parti libéral vaudois indique que les partis politiques dépendent de l'argent de l'économie. Olivier montre [1] que PM versa de 1992 (année avant le vote sur l'initiative jumelle) à 1995 en moyenne 50000 CHF aux partis bourgeois, de l'argent bien investi.

Une étude de la firme R.J. Reynolds (incorporé à JTI) sur le refus de l'initiative jumelle par le peuple indique les méthodes employées par la CISC: «Le lobbying intense: gouvernement, parlement, partis politiques, économie, sport, culture» était décisif, avec le système politique et la coalition protabac [14]. Quelques semaines après son succès, la CISC rencontrait les partis politiques PDC, UDC, Radicaux et libéraux, pour les remercier de «leur engagement dans le vote contre l'initiative jumelle». Les présidents Carlo Schmid, Franz Steinegger (Radicaux) et Hans Uhlmann (UDC) étaient présent, ainsi que les secrétaires des partis Raymond Loretan (PDC) et Philippe Boillod (Libéraux). Le procès verbal constate: "Les membres du Parti radical apprécièrent la présentation faite de l'industrie du tabac en Suisse, car ils n'étaient pas au courant que cette industrie est également une branche exportatrice. Le parti apprécie ce type de contacts et sera prêt à les continuer.» - "A la fin le président de l'UDC propose à l'industrie de s'adresser à lui, en cas de problèmes à résoudre ensemble.» - «Le parti libéral se tient volontiers à disposition de notre industrie, pour aider à résoudre des éventuels problèmes.» «... l'industrie (organise) deux fois par an des réunions avec des parlementaires proches de l'industrie du tabac.»

\section{La prévention du tabagisme dans le Parlement de milice de l'Etat fédéral}

Les politiciens du Parlement de milice refoulent souvent les conflits d'intérêts. Leur attitude loyale person- 
nelle ne les laisse pas prendre conscience qu'ils sont instrumentalisés. De ce fait, dans leur rhétorique libérale sur les «règles liberticides» dominent les formules de relations publiques de l'industrie. Ils oublient que, dans la pensée libérale des Lumières, la liberté des uns autres. Ainsi, dans un texte de l'industrie de 1979 on lit: "Accepter l'initiative c'est confier à la confédération le soin de nous prendre sous tutelle. [Elle] porte atteinte non seulement à la liberté du commerce, mais aussi et surtout à notre liberté individuelle» [15]. En 2012, on retrouve une formule analogue concernant l'initiative pour la protection de la fumée passive: «Une interdiction absolue de fumer serait une atteinte massive de la sphère privée. Les initiateurs veulent placer les citoyennes et citoyens sous tutelle.» L'interdiction de fumer dans l'espace publique fermé est ainsi déformé en interdiction absolue de fumer. A côté de la liberté, le fédéralisme devient un principe à protéger. Ainsi le comité contre l'interdiction absolue de fumer déclare: "Cette initiative va à l'encontre du fédéralisme en imposant aux cantons une règlementation stricte. Il est donc nécessaire de rejeter cette initiative» [16]. Le message «libéral» est répandu par l'alliance de l'économie pour une prévention modérée (AEPM) et par Communication Suisse. Sa directrice commente l'interdiction de la publicité pour protéger les jeunes, en 2018: «L'autorégulation, avec une loi sur les produits du tabac claire, suffit largement. Ce qui va au-delà non seulement empiète sur la liberté du commerce et de la publicité. Pire, avec une interdiction totale, on enlève aux citoyennes et citoyens suisses leur discernement» [17]. Avec cela, elle entretient la confusion en occultant que les produits nicotiniques ne sont pas des aliments, mais des drogues, qui sont un risque particulier justement pour les enfants que l'initiative vise à protéger.

\section{Conclusion}

Dr méd. Rainer M. Kaelin

Plantay 53

CH-1163 Etoy VD

palmier.kaelin[at]bluewin.ch

L'autorégulation de la publicité pour le tabac montre qu'elle est le résultat de l'influence de l'industrie. Il est donc logique d'expliquer par celle-ci également la tac(l'industrie du tabac) ne doit pas causer du tort aux

tique de retardement de traiter la LPTab qui, selon le Conseil des Etats, devra comporter des interdictions de la publicité, alors que les mêmes parlementaires les avaient refusées en bloc dans le premier projet. Ceci, comme les autres mesures alibi de la prévention du tabagisme, montre des représentants du peuple favorables à l'industrie. Qui ont choisi d'ignorer le conflit d'intérêt fondamental entre la santé publique et l'industrie du tabac et qu'une protection de la jeunesse sans interdiction globale de la publicité est impossible. Les réseautages expliquent pourquoi les obligations de la CCLAT ne sont discutées dans notre Parlement que 15 ans après leur mise en œuvre dans d'autres pays.

Les conflits d'intérêts sont inévitables dans un parlement de milice. Sans la volonté politique et l'obligation légale contraignante pour rendre les relations industrie-politique et les flux financiers avec les partis politiques transparents, ils sabordent le processus politique, car ils minent la confiance des citoyens envers l'Etat.

\section{Références}

1 Olivier J. Les fabricants de cigarettes face à la question tabac et santé en Suisse (1962-2003). Thèse de doctorat MD-PhD, Biologie et médecine, UNIL 2019. A appeler comme PDF, www.serval.unil.ch

2 Eichenberger P, Mach A. Organized capital and coordinated market economy. Swiss business interest associations between socio-economic regulation and political influence. In: Mach A, Trampusch C (dir.): Switzerland in Europe. Continuity and change in the Swiss political economy. London, New York: Routledge; 2011, p. 71.

3 Eggly JS. Manque d'humanité et faute politique. M. Corbat quitte l'industrie suisse du tabac, Journal de Genève, $1^{\mathrm{er}}$ avril 1980.

4 Gava R, Varone F, Mach A, Eichenberger S, Christe J, Choa-Blanco C. Interest groups in Parliament: Exploring MP's interest affiliations (2000-2011). Swiss Political Science Review. 2017;23(1):81.

5 British American Tobacco Records. Federal Commission on problems associated with tobacco, 22 mars 1988. Citation dans Olivier J, (1), p. 100-1.

6 Diserens G. Weekly Highlights: week ending June 19, 1992, Switzerland-Finland/Scandinavia-Baltics Duty free. 19 June 1992 pp. 1-2. Philip Morris Records. Citation dans Olivier J, (1), p. 102-3. https://www.industrydocumentslibrary.ucsf.edu/tobacco/doc/ zgkc0118

7 https://www.faire-werbung.ch

8 Kaelin RM. Jugendschutz ohne Werbeverbot? Bull Med Suisses. 2017;98(41):1347-9.

$9 \mathrm{https}: / / \mathrm{ks}-\mathrm{cs} . \mathrm{ch}$

10 www.lch.ch: SER (Syndicat Enseignants Romands) bedauert Entscheid der Lauterkeitskommission (consulté le 31.7.2019).

11 Pantet R. Note sur la séance du 16 novembre 1982 avec MM. Cavadini et Béguin, Conseillers d'Etat, p. 1, Philip Morris Records, clb56e00. Citation dans Olivier J, (1), p. 112-3.

12 Corporate affairs plans, 25 November 1987, p. 6, Philip Morris Records, ity32e00. Citation dans Olivier J, (1), p. 113.

13 Gamboni PV, Albisser G, Rham W. Lettre du parti libéral vaudois à Philip Morris Europe SA, 19 février 1982.

14 Swiss advertising ban case study, November 28, 1993, p. 19 R.J. Reynolds, oim33do0. Citation dans Olivier J, (1), p. 93.

15 Fankhauser W. Good Young Templars' Motion, 23 February 1979. British American Tobacco Records.

16 Arreté fédéral concernant l' initiative populaire Protection contre le tabagisme passif: www.parlament.ch/centers/documents/ fr/11-025-argumentarien-contra-f.pdf

17 Gamper U. Tabakwerbeverbot zum Schutz von Kindern und Jugendlichen? - Dagegen, Zeitlupe 11/2018. 